\title{
Extensive coronary erosion after cocaine consumption treated with branch-per-branch thrombus aspiration
}

\author{
Juan Luis Gutiérrez-Chico \\ Cardiology Department, Campo de Gibraltar Health Trust, Algeciras (Cádiz), Spain
}

A 37-year-old male patient, who was a smoker, presented with chest pain and ST-elevation in inferior leads. He reported cocaine consumption in the preceding 48 hours. The coronary angiogram (44' after onset of symptoms) showed occlusion of the right coronary artery (RCA), with a narrowing in its mid portion (Fig. 1A, asterisk) and contrast retention in the posterior descending (PD) (Fig. 1A, white arrow, Suppl. Video 1). Thrombus aspiration in RCA and PD was performed with an ASAP

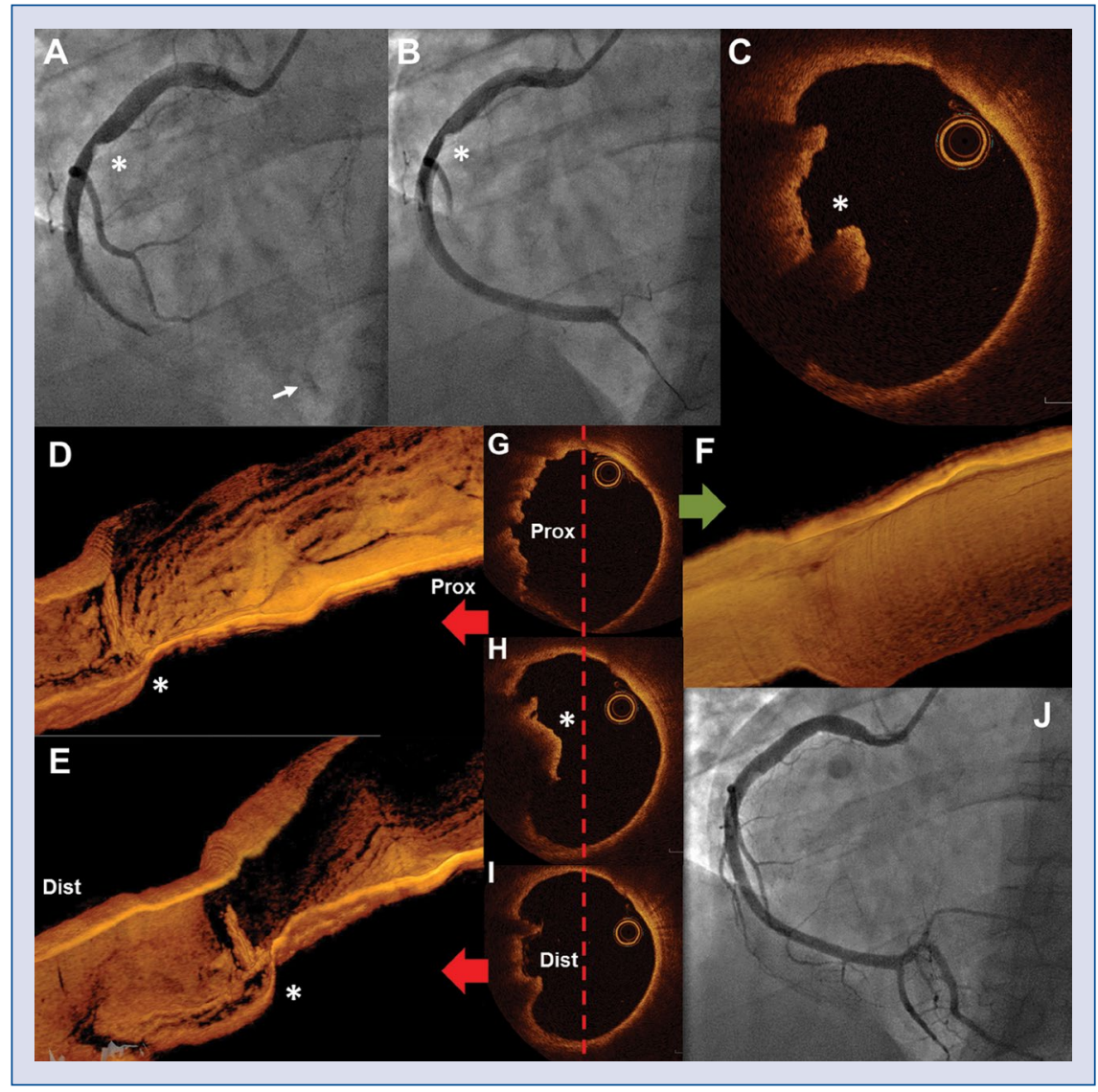

Figure 1. Extensive coronary erosion after cocaine, treated with branch-per-branch thrombus aspiration.

Address for correspondence: Prof. Juan Luis Gutiérrez-Chico, MD, PhD, FESC, FACC, Head of the Cardiology Department, Hospital Punta de Europa, Crtra. Getares s/n, 11207 - Algeciras (Cádiz), Spain, tel: +49 (0) 17630585019 , +34 615 319370, e-mail: juanluis.gutierrezchico@ictra.es

Received: 18.11.2018 Accepted: 9.12.2018 
catheter (Merit Medical, South Jordan, UT), thus restoring Thrombolysis in Myocardial Infarction (TIMI)-3 flow in the PD but not in the atrioventricular artery (Fig. 1B, Suppl. Video 2). Optical coherence tomography showed thrombus remnants at the narrowing (Fig. 1C, asterisk, Suppl. Video 3) in an otherwise non-atherosclerotic, 3-layered vessel without evidence of plaque rupture (Fig. 1C), i.e. an erosion. Three-dimensional image fusion depicted an extensive coronary erosion at the inner curvature of the RCA, with varying amounts of thrombotic burden (Fig. 1D, E), extending from the take-off of the acute marginal to the proximal RCA, in contrast with the smooth appearance of the outer curvature of the same segment (Fig. 1F). Crosssections (Fig. 1G, H, I) show coronary erosion (left side) as opposed to the smooth healthy sectors (right side). The thrombus most likely originated in the large eroded segment, with subsequent distal embolization. Branch-per-branch aspiration was distally performed (Suppl. Video 4) until final TIMI-3 flow was restored in all territories (Fig. 1J, Suppl. Video 5). No stent was implanted. After 7 days of triple antithrombotic therapy an invasive control showed unequivocal regression of the thrombotic burden. The patient was discharged and remains event-free after 3 months of follow-up.

Conflict of interest: None declared 